\title{
Solid pseudopapillary neoplasms of the pancreas are associated with FLI-1 expression, but not with EWS/FLI-1 translocation
}

\author{
Katharina Tiemann, Markus Kosmahl, Julia Ohlendorf, Matthias Krams and \\ Günter Klöppel \\ Department of Pathology, University of Kiel, Kiel, Germany
}

\begin{abstract}
Solid pseudopapillary neoplasms of the pancreas are rare pancreatic tumors with mostly benign behavior, affecting almost exclusively women. Their histogenetic origin is still unsolved, but a recently reported EWS/FLI1 translocation $\mathrm{t}(11 ; 22)(\mathrm{q} 24 ; \mathrm{q} 21)$ and the consistent expression of CD56 and the progesterone receptor, both genes located on the long arm of chromosome 11, point to chromosome 11q as a potential locus of gene aberration in solid pseudopapillary neoplasms. To further elucidate this issue, we studied $\mathbf{3 0}$ cases of solid pseudopapillary neoplasms by comparative genomic hybridization (CGH), fluorescent in situ hybridization (FISH) and immunohistochemistry. Immunohistochemically, 38\% showed nuclear expression of FLI-1 and all cases revealed positivity for CD56 and the progesterone receptor, whereas no solid pseudopapillary neoplasm expressed CD34. No translocation of the EWS gene was found by FISH and no gross chromosomal gain or loss was detected by CGH. It is concluded that FLI-1 expression in solid pseudopapillary neoplasms is not associated with an EWS/FLI-1 translocation. In addition, there are no chromosomal gains or losses, especially on chromosome 11, where the FLI-1 gene is located adjacent to the gene for CD56 (NCAM). These data add another feature to the complex phenotypic appearance of solid pseudopapillary neoplasms.

Modern Pathology (2006) 19, 1409-1413. doi:10.1038/modpathol.3800664; published online 25 August 2006
\end{abstract}

Keywords: solid pseudopapillary neoplasm; FLI-1; CGH; FISH; EWS

Solid pseudopapillary neoplasms of the pancreas have distinctive morphologic features (Figure 1) and show a striking female preponderance. ${ }^{1}$ Ninety to $95 \%$ of these unusual pancreatic neoplasms behave benignly, whereas others are malignant, developing metastases in the mesenterium and the liver. So far, no specific marker for predicting the course of the disease exists, unless the tumor shows clear histological signs of malignancy. ${ }^{2}$

Although a number of studies have been performed, it is still unknown what the direction of differentiation is for the pancreatic solid pseudopapillary neoplasm. Nor do we have much knowledge about genetic changes in solid pseudopapillary neoplasms. At the molecular level, it was recently shown that solid pseudopapillary neoplasms harbor a mutation in exon 3 of the $\beta$-catenin gene, which is

Correspondence: Dr K Tiemann, MD, Department of Pathology, University of Kiel, Michaelisstrasse 11, Kiel 24105, Germany. E-mail: k.peters@path.uni-kiel.de

Received 10 April 2006; revised and accepted 16 June 2006; published online 25 August 2006 associated with aberrant nuclear expression of $\beta$-catenin., ${ }^{3,4}$ Other genetic aberrations of solid pseudopapillary neoplasms include an EWS/FLI-1 translocation $\mathrm{t}(11 ; 22)(\mathrm{q} 24 ; \mathrm{q} 12),{ }^{5}$ a translocation $\mathrm{t}(13 ; 17)(\mathrm{q} 14 ; \mathrm{p} 11),{ }^{6}$ derivatives of chromosomes 1 , 14, and $20^{7}$ and trisomy $3 .^{8}$ A solid pseudopapillary neoplasm investigated by comparative genomic hybridization (CGH) did not show any gross aberrations. ${ }^{9}$

To elucidate the role of a probable EWS/FLI-1 translocation and the involvement of chromosome 11 , we studied 30 well-characterized cases by CGH, fluorescent in situ hybridization (FISH) and with an additional panel of antibodies in order to screen this tumor entity for gross genetic aberrations. With this, we hoped to gain more information about the origin of solid pseudopapillary neoplasms and identify putative predictors of their clinical course.

\section{Materials and methods}

The study was performed on tumor resection specimens from 30 patients with solid pseudopapillary 


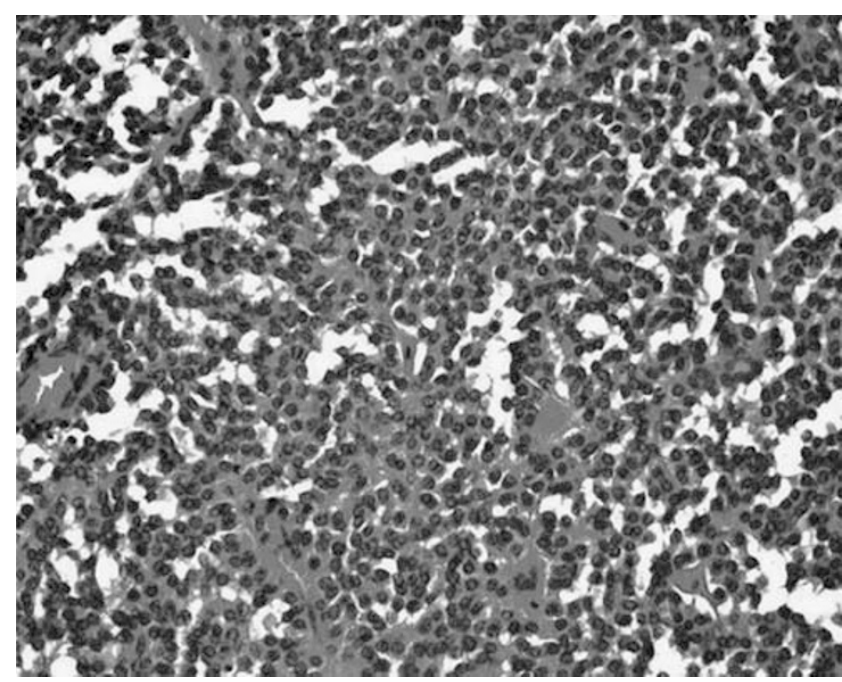

Figure 1 Monomorphic appearance of solid pseudopapillary neoplasm (H\&E).

neoplasm. The cases were retrieved from the files of the Departments of Pathology and Pediatric Pathology, University of Kiel, Germany. Clinical information was obtained from the patients' records. There were 25 female and five male patients. The patients' age ranged from 11 to 63 years, with a mean age of 30 years. One case was malignant, defined by the clinical observation of metastasis. The remaining cases showed the expected benign behavior.

\section{Immunohistochemistry}

For immunohistochemical staining, the following antibodies were used at the stated dilutions: FLI-1 (sc-356, Santa Cruz Biotechnology, Santa Cruz, CA, USA, polyclonal, 1:100); CD34 (1309, Immunotech, Marseille, France, monoclonal, 1:250); NCAM (CD56) (NCL-CD56-1B6, Novocastra, Newcastle upon Tyne, UK, monoclonal, 1:50); $\beta$-catenin (clone CAT-5H10 Zymed, Germany, monoclonal, 1:250); cyclin D1 (clone P2D11F11, Novocastra, 1:10); NSE and synaptophysin (both DakoCytomation, Hamburg, Germany, 1:50) and chromogranin (E001, Linaris, Wertheim-Bettingen, Germany, ready to use). Immunohistochemistry was performed using a standard protocol with heat-induced antigen retrieval in citrate buffer using a pressure cooker, as described previously. ${ }^{10}$ Staining was visualized using the avidin-biotin method with 3,3'-diaminobenzidine as chromogen. Only nuclear staining in at least $10 \%$ of tumor cells was regarded as FLI-1 positivity, the cutoff level for cyclin D1 expression was $5 \%$.

\section{Comparative Genomic Hybridization}

CGH was performed according to a standard protocol, ${ }^{11}$ as described previously. ${ }^{12,13}$ Briefly, a reference $\mathrm{H} \& \mathrm{E}$-stained slide of the tumor block was screened to determine whether the material contained a sufficient number of tumor cells. DNA extraction was carried out according to a routine protocol with a QIAamp DNA Mini Kit (Qiagen, Hilden, Germany). Tumor and control DNA (Vysis, Downers Grove, IL, USA) were labeled by nick translation with biotin-16-dUTP and digoxigenin11-dUTP (Roche Diagnostics, Mannheim, Germany) using DNA polymerase I (Promega, Mannheim, Germany). DNA was digested with DNase I (Roche), adjusted to obtain DNA fragments $500-1000 \mathrm{bp}$ in size. After inactivation of DNase I, unincorporated nucleotides were removed with a QIAquick Nucleotide Removal Kit (Qiagen, Hilden, Germany). For hybridization, $1 \mu \mathrm{g}$ of tumor and reference DNA were cohybridized with $70 \mu \mathrm{g}$ cot1-DNA (Roche) to block repetitive sequences on denatured metaphase slides (Vysis). Hybridization was performed overnight at $37^{\circ} \mathrm{C}$ in a wet chamber. On the following day, the slides were washed four times with $50 \%$ formamide $/ 2 \times \mathrm{SSC}, \mathrm{pH} 7.0$, at $42^{\circ} \mathrm{C}$ and three times in $0.1 \times \mathrm{SSC}$ at $60^{\circ} \mathrm{C}$. Biotin-labeled probes were detected with FITC avidin (Vector Laboratories, Burlingame, CA, USA) and digoxigenin-labeled probes with Cy-3-conjugated anti-digoxigenin (Dianova, Hamburg, Germany). DAPI counterstain was applied for chromosome identification. The slides were covered with antifade solution (Vectashield, Vector Laboratories). Weak FITC signals were amplified with biotinylated anti-avidin D (Vector Laboratories). The slides were screened with a Zeiss Axiophot fluorescence microscope and at least 10 metaphases per case were analyzed with an ISIS analysis system (MetaSystems, Altlussheim, Germany). Ratio values of 1.25 and 0.8 were used as upper and lower thresholds, respectively, for identification of chromosomal gains and losses.

\section{Fluorescence In Situ Hybridization}

FISH was performed on paraffin sections, using the same tissue block, of all cases showing FLI-1 expression. For this, the EWS SpotLight ${ }^{\mathrm{TM}} E W S$ translocation probe pair (84-0300, Zytomed, Berlin, Germany) was used according to the manufacturer's instructions. For evaluation, signals of at least 100 cells were analyzed.

\section{Results}

\section{Histology}

All solid pseudopapillary neoplasms displayed the typical growth pattern with admixed solid and pseudopapillary areas. As signs of regression, areas of necrosis or cholesterol deposits were seen in almost every tumor. The tumor cells were uniform and very rarely showed mitoses (Figure 1). 


\section{Immunohistochemistry}

All solid pseudopapillary neoplasms displayed positivity for CD56 (Figure 2) and negativity for CD34 and chromogranin. Of 29 cases stained for FLI-1, 11 showed nuclear expression (38\%, Figure 3). Eight of the 29 cases $(28 \%)$ were negative for cyclin D1; of these cases, only two showed FLI-1 positivity. Six cases showed synaptophysin expression, all cases were positive for NSE. One case could not be

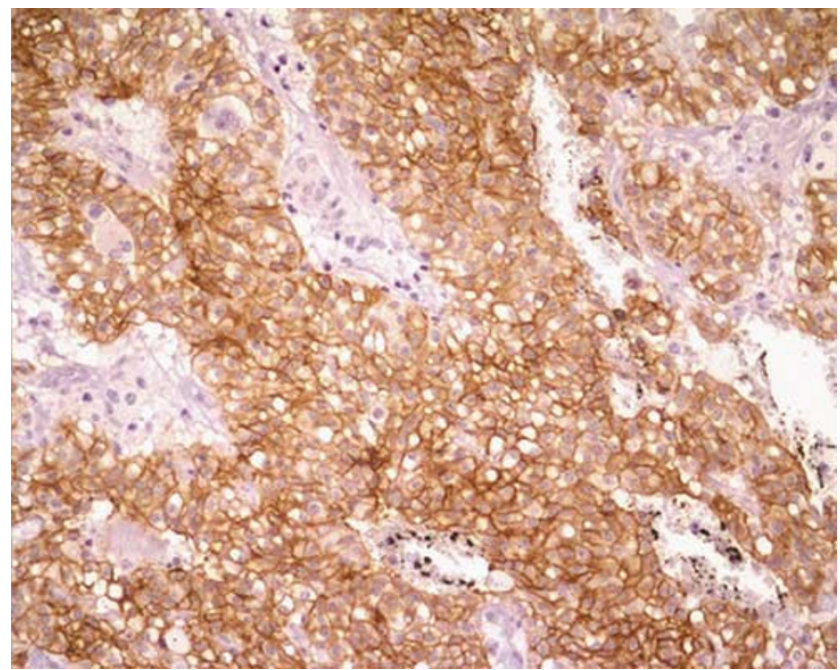

Figure 2 Typical staining pattern for CD56. investigated because no paraffin material from the tumor was left. Table 1 summarizes the immunostaining results.

\section{Comparative Genomic Hybridization}

Twenty-seven of the 30 cases investigated could be evaluated by CGH; the remaining three cases did not yield sufficiently preserved DNA and did not reveal any analyzable hybridization. In the analyzable

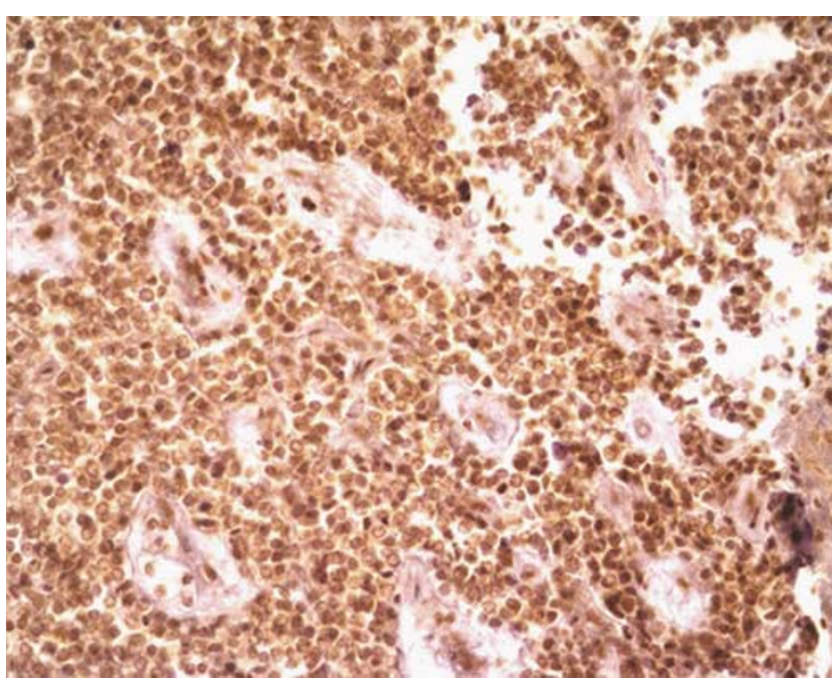

Figure 3 Nuclear expression of FLI-1 in tumor cells of solid pseudopapillary neoplasm.

Table 1 Immunohistochemical profile of solid pseudopapillary neoplasms

\begin{tabular}{|c|c|c|c|c|c|c|c|c|}
\hline Case & $N S E$ & Synaptophysin & Chromogranin & FLI-1 & $\beta$-Catenin & Cyclin D1 & CD34 & CD56 \\
\hline 1 & + & + & - & - & + & + & - & + \\
\hline 3 & + & - & - & - & + & + & - & + \\
\hline 4 & + & - & - & - & + & + & - & + \\
\hline 5 & + & - & - & - & + & + & - & + \\
\hline 6 & + & - & - & - & + & + & - & + \\
\hline 7 & + & - & - & + & + & - & - & + \\
\hline 8 & + & - & - & - & + & - & - & + \\
\hline 9 & + & - & - & - & + & + & - & + \\
\hline 10 & + & - & - & - & + & + & - & + \\
\hline 11 & + & - & - & + & + & + & - & + \\
\hline 12 & + & - & - & - & + & + & - & + \\
\hline 13 & + & + & - & + & + & + & - & + \\
\hline 14 & + & - & - & + & + & + & - & + \\
\hline 15 & + & - & - & - & + & + & - & + \\
\hline 16 & + & + & - & + & + & + & - & + \\
\hline 17 & + & - & - & - & + & - & - & + \\
\hline 18 & + & - & - & + & + & + & - & + \\
\hline 19 & + & - & - & - & + & + & - & + \\
\hline 20 & + & - & - & - & + & + & - & + \\
\hline 21 & + & - & - & + & + & + & - & + \\
\hline 22 & + & - & - & + & + & + & - & + \\
\hline 23 & + & + & - & - & + & - & - & + \\
\hline 24 & + & - & - & + & + & + & - & + \\
\hline 25 & + & - & - & - & + & + & - & + \\
\hline 26 & + & - & - & - & + & - & - & + \\
\hline 27 & + & + & - & - & + & - & - & + \\
\hline 28 & + & - & - & + & + & - & - & + \\
\hline 29 & + & - & - & - & + & - & - & + \\
\hline 30 & + & + & - & + & + & + & - & + \\
\hline
\end{tabular}




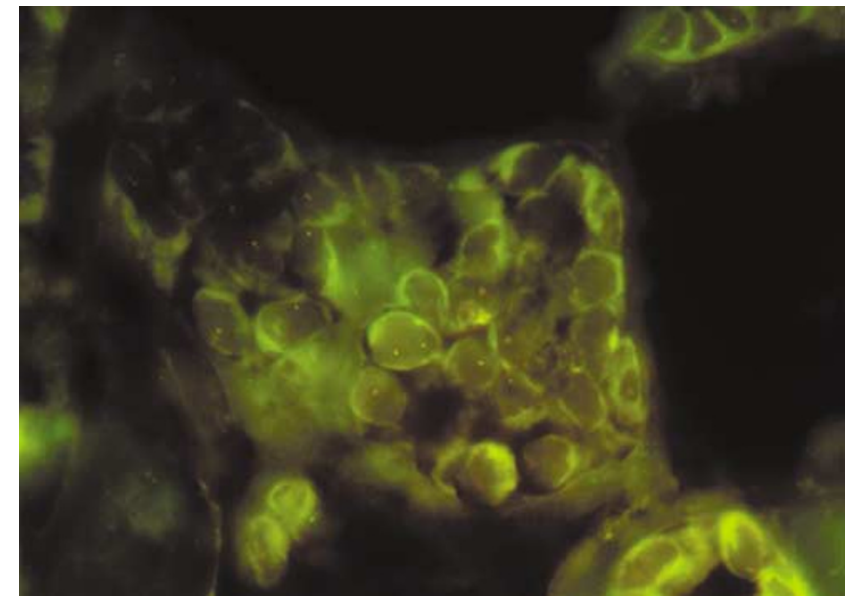

Figure 4 Regular signal distribution without split signals in FISH for the EWS gene.

cases, no chromosomal gains or losses were detected. Especially, no gains were observed at chromosome 11q or chromosome 3. No differences in size or biology were found.

\section{Fluorescence In Situ Hybridization}

The subsequent FISH analysis revealed that all cases that expressed FLI-1 immunohistochemically were negative for EWS translocation (Figure 4).

\section{Discussion}

The genetic and histogenetic background of solid pseudopapillary neoplasms is still an only partially solved mystery. The only known almost consistent genetic aberration is a mutation in exon 3 of the $\beta$-catenin gene. ${ }^{3,4}$ This aberration results in nuclear $\beta$-catenin staining. Functionally, the gene mutation may be responsible for the peculiar morphological growth pattern, which is characterized by pseudopapillary formation owing to the loss of adhesion.

Other chromosomal aberrations have only been studied in a few solid pseudopapillary neoplasms. These comprise an EWS/FLI-1 translocation $\mathrm{t}(11 ; 22)$ (q24;q12), ${ }^{5}$ a translocation $\mathrm{t}(13 ; 17)(\mathrm{q} 14 ; \mathrm{p} 11)^{6}$ and derivatives of chromosomes 1,14 and $20 .{ }^{7}$ Trisomy 3 has also been observed. ${ }^{8}$ We observed nuclear FLI-1 positivity in $38 \%$ of the tumors in our solid pseudopapillary neoplasm series.

The FLI-1 gene and FLI-1 protein are well known for their role in the pathogenesis of Ewing's sarcoma and primitive neuroectodermal tumors (PNET). The majority of these tumors are characterized by the translocation $\mathrm{t}(11 ; 22)(\mathrm{q} 24 ; \mathrm{q} 12)$, resulting in a fusion of the EWS gene on chromosome 22 to the FLI-1 gene on chromosome 11. FLI-1 is a member of the ETS family of transcription factors. It has been characterized as the earliest marker of blood vessels during embryogenesis ${ }^{14}$ and has been shown to be expressed in endothelial and mesodermal tissues. ${ }^{15}$ Its usefulness as a vascular marker has been demonstrated. ${ }^{16}$ Nuclear FLI-1 expression has not been found in normal tissues other than endothelial cells and lymphocytes, but has been detected in Merkel cell carcinoma. ${ }^{17,18}$ The nuclear FLI-1 expression in more than one-third of our solid pseudopapillary neoplasms was not accompanied by positivity for CD34, a well-known vascular marker, nor did FISH results reveal an EWS/FLI-1 translocation, thus contradicting the report of a $\mathrm{t}(11 ; 22)(\mathrm{q} 24 ; \mathrm{q12})$ translocation. $^{5}$ From these data, it can be concluded that solid pseudopapillary neoplasms do not bear any genetic resemblance to Ewing's sarcoma or small blue round cell tumors like PNET.

The case reported in the literature might therefore represent a case of pancreatic PNET or Ewing's sarcoma mistaken for solid pseudopapillary neoplasm, a known differential diagnosis. In the light of our results, positive results for EWS/FLI-1 translocation exclude the diagnosis of solid pseudopapillary neoplasm, adding an additional tool to the diagnostic evaluation of these tumors in the pancreas.

On the other hand, a vascular genesis of solid pseudopapillary neoplasms also seems unlikely, even though FLI-1 is an endothelial marker. In this case, additional expression of other vascular markers should be expected, but as we showed, CD34 expression is missing in solid pseudopapillary neoplasms. Interestingly, the FLI-1 gene lies adjacent to the gene for CD56 (NCAM) on chromosome 11q23-24. CD56 is regularly expressed in solid pseudopapillary neoplasms and is used as a marker of neuroendocrine differentiation. In Merkel cell carcinoma, a neuroendocrine differentiated carcinoma of the skin, FLI-1 expression has been observed, ${ }^{17,18}$ but it was not correlated with prognosis. ${ }^{17}$ In accordance with these findings, FLI-1 expression in solid pseudopapillary neoplasms may be a clue to the histogenesis of these tumors, pointing to a neuroendocrine origin, whereas an origin from endothelial cells seems unlikely, as discussed above. FLI-1 expression was not correlated to the size of solid pseudopapillary neoplasms in our series. Because only one of the solid pseudopapillary neoplasms we investigated was malignant, no conclusions can be drawn concerning its use as a predictive marker in solid pseudopapillary neoplasms.

A report of a solid pseudopapillary neoplasm investigated by $\mathrm{CGH}^{9}$ did not mention any gross aberrations. The latter data are consistent with our study, which did not detect any gains or losses of genetic material. Especially, no gains were observed at chromosome 11q, where the genes for the progesterone receptor, CD56 and FLI-1 lie, or chromosome 3, which harbors the gene locus for $\beta$-catenin. Because of the limitations of the CGH approach, chromosomal translocations and small gains and losses below the threshold of CGH as well 
as gene mutations like the known $\beta$-catenin mutation cannot be detected, although they may exist. Furthermore, there is a conspicuous expression of proteins in solid pseudopapillary neoplasms whose corresponding genes map to chromosome 11q. This includes not only CD56 and FLI-1 but also the progesterone receptor, which is commonly expressed in solid pseudopapillary neoplasms. The event leading to its expression is still unknown, but chromosome $11 \mathrm{q}$ might be involved in a translocation or mutation that leads to the expression of some or all of these three proteins. The missing link between CD56, FLI-1 and the progesterone receptor in solid pseudopapillary neoplasms is the subject of further investigations.

\section{Acknowledgements}

We thank Ms M Pacena, Ms O Batic and Mrs A Bredtmann for their excellent technical assistance.

\section{References}

1 Takahashi Y, Hiraoka N, Onozato K, et al. Solidpseudopapillary neoplasms of the pancreas in men and women: do they differ? Virchows Arch 2006;448: 561-569.

2 Tang LH, Aydin H, Brennan MF, et al. Clinically aggressive solid pseudopapillary tumors of the pancreas. A report of two cases with components of undifferentiated carcinoma and a comparative clinicopathologic analysis of 34 conventional cases. Am J Surg Pathol 2005;29:512-519.

3 Abraham SC, Klimstra DS, Wilentz RE, et al. Solidpseudopapillary tumors of the pancreas are genetically distinct from pancreatic ductal adenocarcinomas and almost always harbor $\beta$-catenin mutations. Am J Pathol 2002;160:1361-1369.

4 Tanaka Y, Kato K, Notohara K, et al. Frequent $\beta$-catenin mutation and cytoplasmic/nuclear accumulation in pancreatic solid-pseudopapillary neoplasm. Cancer Res 2001;61:8401-8404.

5 Maitra A, Weinberg AG, Schneider N, et al. Detection of $t(11 ; 22)(q 24 ; q 12)$ translocation and EWS-FLI-1 fusion transcript in a case of solid pseudopapillary tumor of the pancreas. Pediatr Dev Pathol 2000;3:603-605.
6 Grant LD, Lauwers GY, Meloni AM, et al. Unbalanced chromosomal translocation, $\operatorname{der}(17) \mathrm{t}(13 ; 17)(\mathrm{q} 14 ; \mathrm{p} 11)$ in a solid and cystic papillary epithelial neoplasm of the pancreas. Am J Surg Pathol 1996;20:339-345.

7 Stringer MD, Roberts P, Davison SM, et al. A novel cytogenetic abnormality in a solid and cystic papillary tumour of the pancreas. Med Pediatr Oncol 2003; 41:155-158.

8 Matsubara K, Nigami H, Harigaya H, et al. Chromosome abnormality in solid and cystic tumor of the pancreas. Am J Gastroenterol 1997;92:1219-1221.

9 Tornoczky T, Kalman E, Jakso P, et al. Solid and papillary epithelial neoplasm arising in heterotopic pancreatic tissue of the mesocolon. J Clin Pathol 2001;54:241-245.

10 Kosmahl M, Seada LS, Jänig U, et al. Solid-pseudopapillary tumor of the pancreas: its origin revisited. Virchows Arch 2000;436:473-480.

11 Lichter P, Bentz M, Du Manoir S, et al. Comparative genomic hybridization. In: Verma RS, Babu A (eds). Human Chromosomes: Principles and Techniques, 2nd edn. McGraw-Hill: New York, 1995, pp 191-210.

12 Peters K, Zettl A, Starostik P, et al. Genetic imbalances in primary gastric diffuse large B-cell lymphomas: comparison of comparative genomic hybridization, microsatellite, and cytogenetic analysis. Diagn Mol Pathol 2000;9:58-65.

13 Zettl A, Strobel P, Wagner K, et al. Recurrent genetic aberrations in thymoma and thymic carcinoma. Am J Pathol 2000;157:257-266.

14 Mager AM, Grapin-Botton A, Ladjali K, et al. The avian fli gene is specifically expressed during embryogenesis in a subset of neural crest cells giving rise to mesenchyme. Int J Dev Biol 1998;42:561-572.

15 Vlaeminck-Guillem V, Carrere S, Dewitte F, et al. The Ets family member Erg gene is expressed in mesodermal tissues and neural crests at fundamental steps during mouse embryogenesis. Mech Dev 2000;91: 331-335.

16 Folpe AL, Chand EM, Goldblum JR, et al. Expression of Fli-1, a nuclear transcription factor, distinguishes vascular neoplasms from potential mimics. Am J Surg Pathol 2001;25:1061-1066.

17 Llombart B, Monteagudo C, Lopez-Guerrero JA, et al. Clinicopathological and immunohistochemical analysis of 20 cases of Merkel cell carcinoma in search of prognostic markers. Histopathology 2005;46:622-634.

18 Rossi S, Orvieto E, Furlanetto A, et al. Utility of the immunohistochemical detection of FLI-1 expression in round cell and vascular neoplasm using a monoclonal antibody. Mod Pathol 2004;17:547-552. 